\title{
ACOMPANHAMENTO CLÍNICO FARMACÊUTICO NO CUIDADO AO PACIENTE ADULTO-CIRÚRGICO EM UM HOSPITAL UNIVERSITÁRIO DE PORTO ALEGRE
}

\author{
CLINICAL PHARMACIST FOLLOW-UP IN ADULT \\ SURGICAL PATIENT CARE IN A UNIVERSITY HOSPITAL OF \\ PORTO ALEGRE
}

\author{
Caroline Tortato ${ }^{1}$, Paola Hoff Alves ${ }^{1}$, Carlos Alberto Yasin Wayhs ${ }^{1}$
}

\begin{abstract}
RESUMO
Introdução: O farmacêutico clínico já está bem estabelecido em algumas instituições e muitos serviços de saúde contam com este profissional em suas equipes, entretanto, poucos conseguem sistematizar o seu trabalho e mapear os dados das atividades desenvolvidas, demonstrando a relevância do profissional na equipe de saúde. O objetivo deste trabalho foi avaliar o acompanhamento clínico-farmacêutico em uma unidade de internação adulto-cirúrgica em um hospital universitário de Porto Alegre.
\end{abstract}

Métodos: Estudo descritivo retrospectivo que quantificou as principais atividades do farmacêutico clínico em uma unidade de internação adulto-cirúrgica no período de janeiro a maio de 2019. Este projeto foi aprovado no Comitê de Ética em Pesquisa da referida instituição.

Resultados: 859 pacientes foram admitidos na unidade cirúrgica avaliada, dos quais 490 foram revisados pelo farmacêutico na admissão hospitalar, correspondendo à taxa média de $57,27 \%$. A taxa média de conciliação medicamentosa realizada foi de $14,83 \%$, totalizando 73 pacientes conciliados por entrevista. 361 intervenções farmacêuticas foram realizadas no período estudado, sendo 54 relacionadas a conciliação medicamentosa, com o número total de adesões de 232. As principais especialidades cirúrgicas que internam pacientes na unidade em questão foram a Ortopedia, Cirurgia do Aparelho Digestivo, Urologia e Cirurgia Vascular.

Conclusões: Foi possível avaliar o acompanhamento clínico farmacêutico em uma unidade de internação adulto-cirúrgica em um hospital universitário de Porto Alegre, através da quantificação das taxas de pacientes revisados e de conciliação medicamentosa, do número de intervenções farmacêuticas e suas adesões, além de caracterizar as principais especialidades médicas cirúrgicas envolvidas.

Palavras-chave: Farmácia clínica; Pacientes internados; Intervenções farmacêuticas

\section{ABSTRACT}

Introduction: Clinical pharmacists are already well established in some institutions, and many health services have these professionals in their teams. However, few are able to systematize their work and map data from the developed activities, demonstrating the relevance of these professionals in the health team. This study aimed to evaluate the clinical pharmacist follow-up in an adult surgical inpatient unit in a university hospital in Porto Alegre.

Methods: This is a retrospective, descriptive study that quantified the main activities of the clinical pharmacist in an adult surgical inpatient unit from January to May 2019. This project was approved by the Research Ethics Committee of the institution.
Clin Biomed Res. 2021;41(4):1-7

1 Serviço de Farmácia do Hospital de Clínicas de Porto Alegre, Universidade Federal do Rio Grande do Sul. Porto Alegre, RS, Brasil.

Autor correspondente: Caroline Tortato ctortato@hcpa.edu.br Serviço de Farmácia do Hospital de Clínicas de Porto Alegre, Universidade Federal do Rio Grande do Sul

Rua Ramiro Barcelos, 2350 90035-903, Porto Alegre, RS, Brasil. 
Tortato et al.

Results: Of 859 patients admitted to the surgical unit, 490 were reviewed by the pharmacist on hospital admission, corresponding to an average rate of $57.27 \%$. The average medication reconciliation rate was $14.83 \%$, totaling 73 patients reconciled per interview. Of 361 pharmaceutical interventions performed during the study period, 54 were related to medication reconciliation, and the total number of adhesions was 232 . The main surgical specialties associated with admission to the study unit were Orthopedics, Digestive System Surgery, Urology, and Vascular Surgery.

Conclusions: It was possible to evaluate the clinical pharmacist follow-up in an adult surgical inpatient unit in a university hospital in Porto Alegre by quantifying the rates of reviewed patients and medication reconciliations as well as the number of pharmaceutical interventions and their adherences, in addition to characterizing the main medical-surgical specialties involved.

Keywords: Clinical pharmacy; Inpatients; Pharmaceutical interventions

\section{INTRODUÇÃO}

De acordo com a American College of Clinical Pharmacy, a farmácia clínica é definida como a disciplina na área da saúde em que o farmacêutico presta assistência ao paciente por meio da prática do uso racional de medicamentos com a adequação da terapia medicamentosa e da prevenção de doenças ${ }^{1}$. No entanto, não existe um consenso sobre a definição de "farmácia clínica", embora todas as definições propostas refiram-se à contribuição dos farmacêuticos para uma terapia medicamentosa racional e de alta qualidade ${ }^{2}$.

O termo "farmácia clínica" foi criado para descrever o trabalho dos farmacêuticos, cujas principais atividades são interagir com a equipe de saúde, avaliar e monitorar a resposta terapêutica dos pacientes, realizar intervenções, recomendações e fornecer informações sobre medicamentos ${ }^{3}$. O farmacêutico clínico trabalha promovendo a saúde utilizando diretrizes terapêuticas baseadas em evidências, contribuindo e intervindo na prescrição médica sob aspectos técnicos e econômicos, para a obtenção de melhores resultados clínicos ao paciente ${ }^{3-6}$.

A avaliação ou análise da prescrição médica consiste em verificar o tipo de medicamento prescrito, se a indicação está correta, se as doses e frequências estão adequadas, se a via de administração está correta, as possíveis interações medicamentosas de relevância clínica e possíveis reações adversas que o paciente pode apresentar ${ }^{7}$. Ações simples de serem implantadas, como análise de prescrições, são capazes de identificar e prevenir problemas relacionados a medicamentos, evitar perdas financeiras e agregar imensurável valor na segurança do paciente ${ }^{8}$. Portanto, revisar e validar a prescrição medicamentosa é um passo imprescindível para atender aos requisitos de qualidade no uso de medicamentos ${ }^{9}$.

Além de avaliar a prescrição médica, é necessário avaliar os exames laboratoriais dos pacientes, as evoluções e registros feitos pela equipe multiprofissional envolvida no cuidado e, também, compartilhar informações importantes sobre medicamentos ${ }^{7}$. Neste cenário de informações sobre medicamentos, a conciliação medicamentosa possui papel fundamental. Ela objetiva reduzir erros de medicação resultantes de discrepâncias na prescrição, principalmente quando o paciente transita por distintos serviços de saúde ou diferentes níveis de atenção dentro da mesma instituição de saúde ${ }^{10}$.

A conciliação medicamentosa caracteriza-se pela elaboração de uma lista precisa de todos os medicamentos utilizados pelo paciente (nome, concentração, forma farmacêutica, dose, via frequência, duração do tratamento), conciliando as informações do prontuário, da prescrição, do paciente ou de cuidadores ${ }^{10}$. Foi demonstrado que a realização da conciliação medicamentosa no momento da admissão do paciente reduz o número de discrepâncias entre os medicamentos utilizados previamente pelo paciente e os prescritos durante a internação. Até $27 \%$ de todos os erros de prescrição podem ser relacionados ao histórico de medicamentos incompletos, sendo que 60 a $70 \%$ desses históricos apresentam pelo menos um erro ${ }^{11}$.

A intervenção farmacêutica, segundo o Consenso Brasileiro de Atenção Farmacêutica de 2002, "é um ato planejado, documentado e realizado junto ao usuário e aos profissionais de saúde, que visa resolver ou prevenir problemas que interferem ou podem interferir na farmacoterapia, sendo parte integrante do processo de acompanhamento/seguimento farmacoterapêutico"12. Neste contexto, para que haja a adesão de médicos às intervenções realizadas pelo profissional farmacêutico, é preciso confiança entre estes profissionais, além do trabalho desenvolvido em conjunto, unindo-se os diferentes conhecimentos para aperfeiçoar a assistência ao paciente ${ }^{7}$.

Além das atividades já citadas, o papel do farmacêutico clínico inclui a educação do paciente e familiares, garantindo que estes compreendam o tratamento e tenham adesão a ele. A orientação farmacêutica pode ser realizada desde o ingresso do paciente na instituição até a alta hospitalar, sendo possível que o farmacêutico seja requisitado para dar esclarecimentos ao paciente ou familiar sobre quaisquer dúvidas relativas ao seu tratamento farmacoterapêutico ${ }^{9}$.

A regulamentação das atribuições clínicas do farmacêutico no Brasil ocorreu através da Resolução $n^{\circ} 585$ de 29 de agosto de 2013, publicada pelo Conselho Federal de Farmácia. As atribuições clínicas regulamentadas pela presente resolução constituem prerrogativa do farmacêutico legalmente 
habilitado e registrado no Conselho Regional de Farmácia de sua jurisdição. As atribuições dispostas nesta resolução correspondem aos direitos, responsabilidades e competências do farmacêutico no desenvolvimento das atividades clínicas e na provisão de serviços farmacêuticos ${ }^{13}$.

Diante deste cenário, o objetivo deste trabalho foi avaliar o acompanhamento clínico farmacêutico em uma unidade de internação adulto-cirúrgica em um hospital universitário de Porto Alegre.

\section{MÉTODOS}

Trata-se de um estudo transversal, de caráter descritivo e retrospectivo, realizado através das análises de dados do acompanhamento clínico farmacêutico nos meses de janeiro a maio de 2019, em uma unidade de internação adulto-cirúrgica em um hospital universitário de Porto Alegre. Esta instituição de saúde é considerada de alta complexidade e é certificada pela Acreditação Internacional da Joint Commission International (JCl). Possui aproximadamente 850 leitos de internação, dos quais 45 pertencem à unidade em questão.

A seção de Farmácia Clínica deste hospital universitário realiza o seu trabalho com base no Bundle Farmacêutico, instrumento desenvolvido internamente para o acompanhamento hospitalar do farmacêutico clínico e avaliação de seus resultados. A revisão do paciente consiste na avaliação da prescrição médica, revisão das evoluções da equipe multiprofissional no prontuário eletrônico, revisão dos exames laboratoriais, sinais vitais e realização da conciliação medicamentosa, quando os pacientes atenderem aos critérios de inclusão para tal atividade.

Os critérios para a realização da conciliação medicamentosa na admissão são: pacientes com fibrose cística, HIV positivo e pacientes acompanhados por equipe, tais como Comanejo-Ortopedia, Transplantes,
Medicina Interna, dentre outras. Nestes casos, a conciliação é realizada através de entrevista com paciente ou familiar/cuidador. O farmacêutico clínico possui um prazo de 72 horas a partir da internação do paciente na unidade para realização da conciliação medicamentosa na admissão.

Nesse contexto, foram avaliados os seguintes indicadores de processo: 1) Taxa de pacientes revisados; 2) Taxa de conciliação medicamentosa; 3) Número de intervenções farmacêuticas, 4) Taxa de adesão às intervenções farmacêuticas e 5) Frequência de internações por especialidade Médica Cirúrgica. Os dados foram coletados no Microsoft Office Excel $2013^{\circledR}$, por dois farmacêuticos clínicos que atuavam na unidade de internação adulto-cirúrgica em questão, sendo um farmacêutico clínico contratado não exclusivo e um residente de farmácia do programa Atenção Integral ao Paciente Adulto Cirúrgico. Este trabalho foi aprovado no Comitê de Ética em Pesquisa da referida instituição sob o número 2019-0408.

\section{RESULTADOS E DISCUSSÃO}

A figura 1 evidencia a taxa de pacientes revisados na unidade de internação adulto-cirúrgica no período de janeiro a maio de 2019 pelo farmacêutico clínico. Durante o período do estudo, foram admitidos na unidade cirúrgica avaliada um total de 859 pacientes, dos quais 490 foram revisados pelo farmacêutico na admissão hospitalar, correspondendo à taxa média de $57,27 \%$ de pacientes revisados. Este resultado demonstrou-se superior quando comparado ao atendimento prestado pelo serviço de farmácia de uma instituição pública federal que é referência nacional para cirurgias de alta complexidade em ortopedia, no Rio de Janeiro, que atendeu 13,6\% (747/5476) dos pacientes que internaram na instituição ${ }^{14}$.

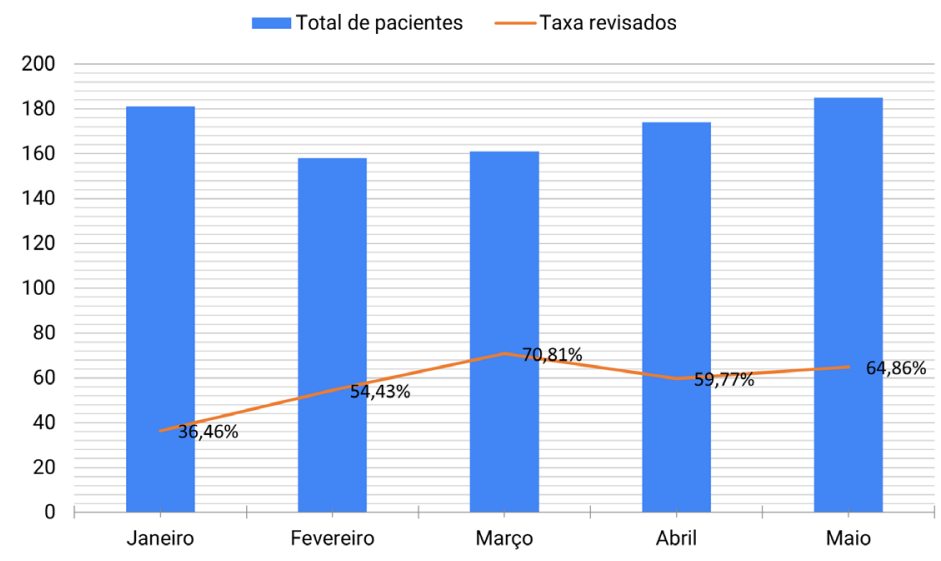

Figura 1: Taxa de pacientes revisados por farmacêuticos clínicos em unidade de internação adulto-cirúrgica em hospital universitário de Porto Alegre, janeiro-maio/2019. 
No período da realização do estudo em questão, a unidade contava com o acompanhamento de dois farmacêuticos clínicos, sendo um farmacêutico clínico contratado não exclusivo e um residente de farmácia do programa Atenção Integral ao Paciente Adulto Cirúrgico. Foi possível perceber uma taxa de pacientes revisados menor nos dois primeiros meses do ano (janeiro e fevereiro), provavelmente atribuída ao período de férias destes profissionais e estágios optativos do residente em outras instituições de saúde. Também, foi possível identificar que, em média, cada profissional farmacêutico consegue revisar aproximadamente $30 \%$ dos pacientes novos admitidos na unidade de internação em questão. Sendo assim, para viabilizar a revisão de um número maior de pacientes, aproximando-se aos $100 \%$, seria necessário mais um farmacêutico clínico desempenhando estas atividades.

Através da figura 2, foi possível verificar que a taxa média de conciliação medicamentosa realizada pelo farmacêutico clínico nesta unidade no período de janeiro a maio de 2019 foi de $14,83 \%$, totalizando 73 pacientes conciliados por entrevista. Este resultado demonstra que, comparado ao número total de pacientes internados, a taxa de conciliação medicamentosa é relativamente baixa. Entretanto, esta taxa mostrou-se superior ao critério de conciliação utilizado na Seção de Farmácia Clínica da referida instituição para a unidade de internação adulto-cirúrgica em questão, onde é prioritária em pacientes com fibrose cística, HIV positivo e naqueles pacientes acompanhados por equipe, tais como Comanejo-Ortopedia, Transplantes, Medicina Interna, dentre outras. No período avaliado, apenas 36 pacientes $(7,35 \%)$ atenderam a estes critérios de conciliação prioritária, demonstrando que os critérios para a conciliação medicamentosa na admissão do paciente já mencionados são muito específicos e pouco sensíveis para o perfil de pacientes admitidos nesta unidade.

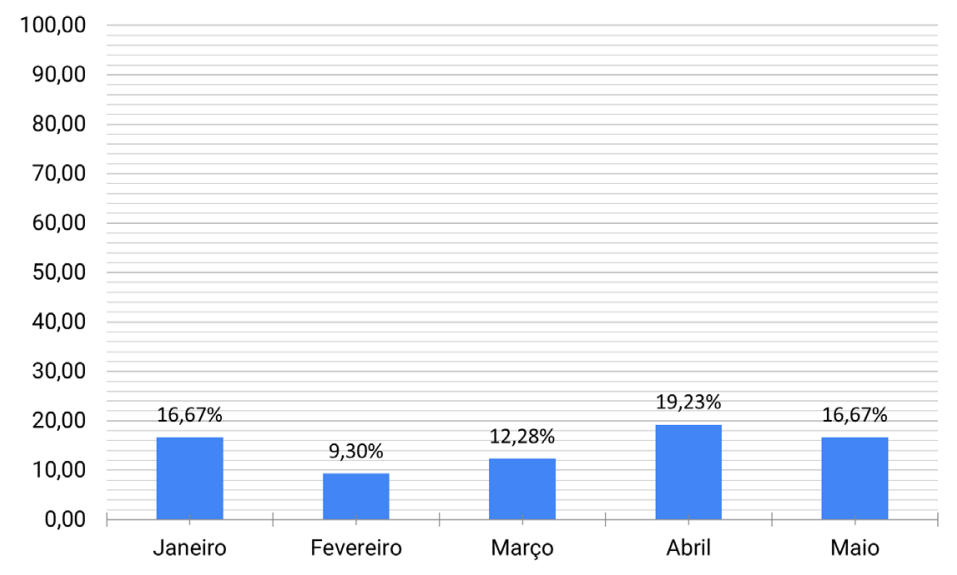

Figura 2: Taxa de conciliação medicamentosa realizada por farmacêuticos clínicos em unidade de internação adultocirúrgica em hospital universitário de Porto Alegre, janeiro-maio/2019.

Nesse sentido, evidência de um estudo prospectivo realizado em pacientes idosos de um hospital terciário na Alemanha apresentou uma taxa de conciliação medicamentosa realizada pelos farmacêuticos, na admissão, de $61,80 \%$ dos pacientes internados no referido hospital, durante o período do estudo ${ }^{15}$. Comparativamente, esta taxa é muito superior ao resultado apresentado por nós, corroborando a necessidade de revisão dos critérios para a conciliação medicamentosa na admissão do paciente em nosso serviço. Entretanto, salienta-se que no estudo alemão, a conciliação medicamentosa não necessariamente foi realizada através de entrevista com paciente ou familiar, conforme executado em nosso estudo.

Além disso, estudos comparando diferentes práticas de conciliação medicamentosa para pacientes internados e seus efeitos sobre os resultados clínicos são escassos na literatura científica internacional ${ }^{16}$. No entanto, evidências disponíveis sustentam que as intervenções de conciliação medicamentosa realizadas pelas equipes da farmácia clínica de diversas instituições se concentram em pacientes com alto risco de eventos adversos, reduzindo estes potenciais riscos e trazendo benefícios à segurança do paciente ${ }^{16-20}$. Contudo, estudos de alta qualidade como ensaios clínicos randomizados são necessários para determinar as abordagens mais eficazes para a execução da conciliação medicamentosa em pacientes internados e a verificação de seus efeitos sobre os resultados clínicos na prática, uma vez que estudos transversais, de caráter descritivo e retrospectivo, como o apresentado aqui, possuem 
limitações para tais conclusões. Ainda, este estudo apresenta limitações relacionadas aos registros da Seção de Farmácia Clínica, uma vez que os dados foram obtidos por dois profissionais diferentes.

As intervenções realizadas pelos farmacêuticos clínicos deste hospital são classificadas entre os seguintes tipos: conciliação medicamentosa, farmacovigilância, interações medicamentosas/incompatibilidade, protocolos institucionais (anticoagulação oral, profilaxia cirúrgica, delirium, por exemplo), medicamentos de urgência/emergência, padronização do preparo de medicamentos, sustentabilidade econômica, ajuste de dose conforme taxa de filtração glomerular, vancocinemia e outros. O número de intervenções farmacêuticas e suas adesões foram apresentados na figura 3. Foram realizadas 361 intervenções farmacêuticas no período estudado, sendo 54 relacionadas a conciliação medicamentosa, com o número total de adesões de 232 . Foi possível observar que, em média, $64,27 \%$ do número de intervenções foi aderido pelo prescritor. Um estudo realizado em um hospital filantrópico de Porto Alegre no período de outubro de 2017 a março 2018 avaliou a implementação da conciliação medicamentosa através dos registros de prontuário. Em um total de 1379 pacientes, 347 (25\%) foram conciliados pelo farmacêutico, sendo necessária intervenção junto a equipe médica em $30 \%$ dos casos $^{21}$.

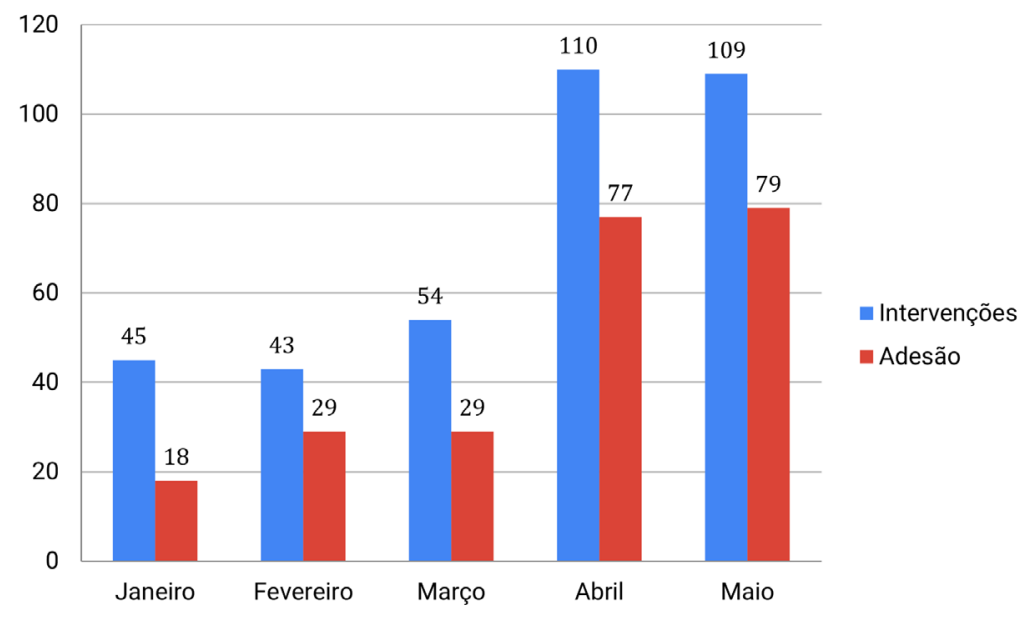

Figura 3: Número de intervenções farmacêuticas realizada por farmacêuticos clínicos em unidade de internação adultocirúrgica em hospital universitário de Porto Alegre e adesões, janeiro-maio/2019.

Os dados apresentados vão ao encontro da literatura nacional, evidenciando uma taxa de adesão do corpo clínico às intervenções farmacêuticas na prescrição de pacientes hospitalizados variando bastante entre os centros de saúde. Em um estudo realizado no Hospital de Clínicas da Universidade Federal do Paraná (HCUFPR), por exemplo, a aceitação das intervenções feitas no período de julho de 2011 a julho de 2012, nas Unidades de Terapia Intensiva para Adultos foi de $76,32 \%$ $(74,71 \% \text { aceitos e } 1,61 \% \text { aceitos com alterações })^{22}$. Já no Hospital Israelita Albert Einstein, a adesão do corpo clínico às intervenções do farmacêutico saiu de $93,35 \%$ em 2003 para 99,30\% em 2010, evidenciando o reconhecimento por parte dos médicos do trabalho desempenhado pelo farmacêutico clínico, assim como da importância deste profissional no processo de segurança de toda a cadeia de medicamentos ${ }^{7}$. Este último caso, serve de referência às demais instituições de saúde, uma vez que possui uma experiência bemsucedida na implantação do serviço de farmácia clínica.
Além disso, a população pós cirúrgica ortopédica foi a mais prevalente em um processo de conciliação medicamentosa em estudo conduzido em um Hospital Universitário do Paraná. Do total de pacientes conciliados, $62,5 \%$ necessitavam de alguma intervenção farmacêutica ${ }^{23}$. Os dados apresentados no presente estudo evidenciaram uma necessidade de intervenção farmacêutica em $74 \%$ dos pacientes conciliados, o que corrobora com dados já publicados e reflete a necessidade de um melhor acompanhamento farmacêutico nas especialidades cirúrgicas, principalmente na avaliação da necessidade de conciliação dos medicamentos de uso prévio, uma vez que muitos pacientes não são acompanhados pela equipe cirúrgica previamente ao procedimento.

Em relação ao cenário internacional, estudo retrospectivo realizado na enfermaria geriátrica de um hospital universitário da Turquia evidenciou que 329 intervenções foram recomendadas pelo farmacêutico, sendo que $85,4 \%$ dessas foram aceitas 
pelo médico assistente ${ }^{24}$. Resultados comparáveis foram encontrados na Bélgica onde um total de 1.066 intervenções farmacêuticas foram elaboradas no período de 7 meses, sendo que a taxa de aceitação pelos médicos foi de $87,8 \%{ }^{25}$.

$\mathrm{Na}$ figura 4, foi possível verificar que as principais especialidades cirúrgicas que internam pacientes na unidade em questão foram a Ortopedia (ORT), seguida pela Cirurgia do Aparelho Digestivo (CAD), Urologia (URL) e Cirurgia Vascular (CIV). Cabe ressaltar, que esta unidade possui leitos reservados para atender aos pacientes pré e pós-cirúrgicos da especialidade Ortopedia, o que não ocorre em outras unidades da mesma instituição. A referida especialidade foi escolhida para ter um atendimento diferenciado do farmacêutico clínico em virtude da alta demanda de pacientes e de sua complexidade, exigindo a sua participação em rounds multidisciplinares.

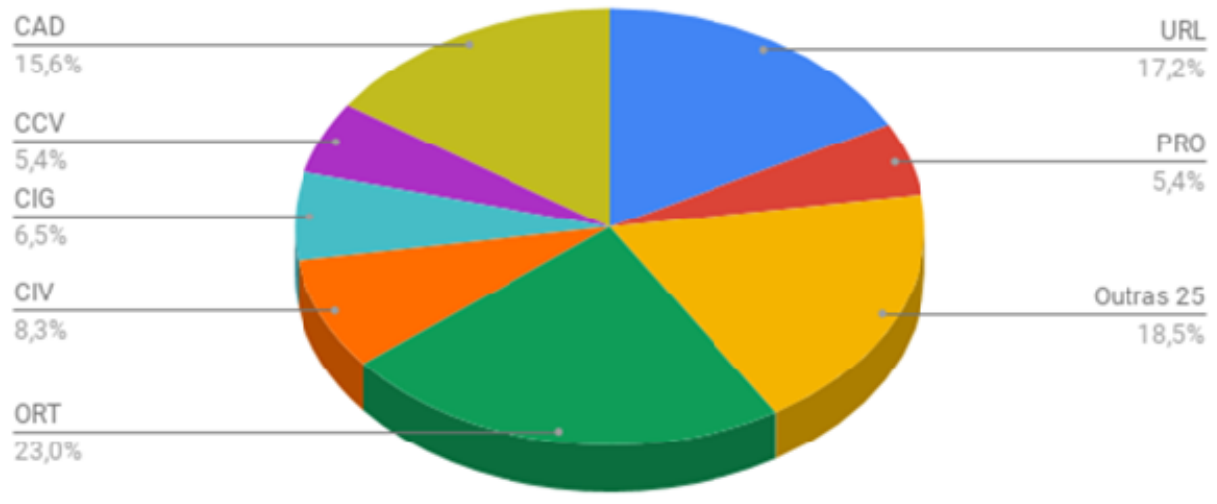

Figura 4: Especialidades cirúrgicas em unidade de internação adulto-cirúrgica em hospital universitário de Porto Alegre, janeiro-maio/2019: CAD - Cirurgia do aparelho abdominal; CCV - Cardiovascular; CIG - Geral; CIV - Vascular; ORT Ortopedia; URL - Urologia; PRO - Proctologia.

Através deste trabalho foi possível avaliar o acompanhamento clínico farmacêutico em uma unidade de internação adulto-cirúrgica em um hospital universitário de Porto Alegre, através da quantificação das taxas de pacientes revisados e de conciliação medicamentosa, do número de intervenções farmacêuticas e suas adesões, além de caracterizar as principais especialidades médicas cirúrgicas envolvidas. Foi possível concluir que para viabilizar a revisão de um número maior de pacientes na unidade de internação supracitada, aproximandose aos $100 \%$, seria necessário mais um farmacêutico clínico desempenhando esta atividade. Os atuais critérios utilizados para a conciliação medicamentosa na admissão dos pacientes são muito específicos e pouco sensíveis para o perfil desta unidade de internação, o que pode indicar uma necessidade de revisão destes critérios pela Seção de Farmácia Clínica da instituição. Além disso, evidenciou-se a necessidade de um aumento na taxa de adesão às intervenções farmacêuticas, corroborando com maior segurança ao paciente. Ainda, foi possível verificar que as principais especialidades cirúrgicas que internam pacientes na unidade em questão foram Ortopedia, Cirurgia do Aparelho Digestivo, Urologia e Cirurgia Vascular. Por fim, entende-se a grande necessidade de monitoramento e acompanhamento do farmacêutico clínico nas especialidades cirúrgicas, visto que na grande maioria das vezes a equipe não conhece previamente o histórico de medicamentos do paciente, sendo o primeiro contato na avaliação pré-anestésica ou, até mesmo, já no bloco cirúrgico. Além disso, o farmacêutico pode contribuir em outros cuidados, os quais não foram mensurados neste trabalho, como por exemplo na otimização da dose e tempo da profilaxia antimicrobiana, interações medicamentosas, prevenção e manejo de tromboembolismo venoso, dentre outras.

\section{Conflitos de Interesse}

Os autores declaram não ter nenhum conflito de interesse. 


\section{REFERÊNCIAS}

1. American College of Clinical Pharmacy. A vision of pharmacy's future roles, responsibilities and manpower needs in the United States. Pharmacotherapy. 2000;20(8):991-1020.

2. De Rijdt T, Willems L, Simoens $S$. Economic effects of clinical pharmacy interventions: a literature review. Am J Health Syst Pharm. 2008;65(12):1161-72.

3. Santos L, Torriani MS, Barros E, editores. Medicamentos na prática da farmácia clínica. Porto Alegre: Artmed; 2013.

4. Arenas-Villafranca JJ, RodríguezCamacho JM, Pérez-Moreno MA, Moreno-Santamaría M, MartosPérez FA, Tortajada-Goitia B. The role of clinical pharmacists in the optimisation of medication prescription and reconciliation on admission in an emergency department. Eur J Hosp Pharm. 2018;25(e1):e59-61.

5. Cab Bond CA, Raehl CL. Clinical and economic outcomes of pharmacistmanaged antimicrobial prophylaxis in surgical patients. Am J Health Syst Pharm. 2007;64(18):1935-42.

6. Arantes T, Durval CC, Pinto VB. Avaliação da economia gerada por meio das intervenções farmacêuticas realizadas em um hospital universitário terciário de grande porte. Clin Biomed Res. 2020;40(2):96-104.

7. Ferracini FT, Borges Filho WM. Farmácia Clínica: Segurança na Prática Hospitalar. São Paulo: Atheneu; 2011.

8. Aguiar KS, Santos JM, Cambrussi MC, Picolotto S, Carneiro MB. Patient safety and the value of pharmaceutical intervention in a cancer hospital. Einstein (Sao Paulo). 2018;16(1):eAO4122.

9. Sociedade Brasileira de Farmácia Hospitalar e Serviços de Saúde. Guia de boas práticas em farmácia hospitalar e serviços de saúde. São Paulo: Ateliê Vide o Verso; 2009.
10. Conselho Federal de Farmácia (BR). Serviços farmacêuticos diretamente destinados ao paciente, à família e à comunidade: contextualização e arcabouço conceitual. Brasília, DF; 2016.

11. Lombardi NF, Mendes AEM, Lucchetta RC, Reis WCT, Fávero MLD, Correr CJ. Análise das discrepâncias encontradas durante a conciliação medicamentosa na admissão de pacientes em unidades de cardiologia: um estudo descritivo. Rev Lat Am Enfermagem. 2016;24:e2760.

12. Finatto RB, Caon S, Bueno D. Intervenção farmacêutica como indicador de qualidade da assistência hospitalar. Rev Bras Farm. 2012;93(3):364-70.

13. Conselho Federal de Farmácia (BR). Resolução $n^{\circ} 585$, de 29 de agosto de 2013: regulamenta as atribuições clínicas do farmacêutico e dá outras providências. Brasília, DF; 2013.

14. Nunes PHC, Pereira BMG, Nominato JCS, Albuquerque EM, Silva LFN, Castro IRS, et al. Intervenção farmacêutica e prevenção de eventos adversos. Rev Bras Cienc Farm. 2008;44(4):691-9.

15. Cortejoso L, Dietz RA, Hofmann G, Gosch M, Sattler Z. Impact of pharmacist interventions in older patients: a prospective study in a tertiary hospital in Germany. Clin Interv Aging. 2016;11:1343-50.

16. Mueller SK, Sponsler KC, Kripalani S, Schnipper JL. Hospital-based medication reconciliation practices: a systematic review. Arch Intern Med. 2012;172(14):1057-69.

17. Mekonnen AB, McLachlan AJ, Brien JE. Effectiveness of pharmacist-led medication reconciliation programmes on clinical outcomes at hospital transitions: a systematic review and meta-analysis. BMJ Open. 2016;6(2):e010003.
18. Galvin M, Jago-Byrne MC, Fitzsimons M, Grimes T. Clinical pharmacist's contribution to medication reconciliation on admission to hospital in Ireland. Int J Clin Pharm. 2013;35(1):14-21.

19. Eisenhower C. Impact of pharmacistconducted medication reconciliation at discharge on readmissions of elderly patients with COPD. Ann Pharmacother. 2014;48(2):203-8.

20. Redmond P, Grimes TC, McDonnell R, Boland F, Hughes C, Fahey T. Impact of medication reconciliation for improving transitions of care. Cochrane Database Syst Rev. 2018;8(8):CD010791.

21. Santos CO, Lazaretto FZ, Lima LH, Azambuja MS, Millão LF. Reconciliação de medicamentos: processo de implantação em um complexo hospitalar com a utilização de sistema eletrônico. Saude Debate. 2019;43(121):368-77.

22. Reis WCT, Scopel CT, Correr CJ, Andrzejevski VMS. Análise das intervenções de farmacêuticos clínicos em um hospital de ensino terciário do Brasil. Einstein (Sao Paulo). 2013;11(2):190-6.

23. Frizon F, Santos AH, Caldeira LF, Menolli PVS. Reconciliação de medicamentos em hospital universitário. Rev Enferm UERJ. 2014;22(4):454-60.

24. Ertuna E, Arun MZ, Ay S, Koçak FÖK, Gökdemir B, İspirli G. Evaluation of pharmacist interventions and commonly used medications in the geriatric ward of a teaching hospital in Turkey: a retrospective study. Clin Interv Aging. 2019;14:587-600.

25. Spinewine A, Dhillon S, Mallet L, Tulkens PM, Wilmotte L, Swine C. Implementation of ward-based clinical pharmacy services in Belgium: description of the impact on a geriatric unit. Ann Pharmacother. 2006;40(4):720-8. 